\title{
Quantum Chaos Border for Quantum Computing
}

\author{
B. Georgeot and D. L. Shepelyansky \\ Laboratoire de Physique Quantique, UMR 5626 du CNRS, Université Paul Sabatier, F-31062 Toulouse Cedex 4, France
}

(October 19, 1999; revised January 17, 2000)

\begin{abstract}
We study a generic model of quantum computer, composed of many qubits coupled by short-range interaction. Above a critical interqubit coupling strength, quantum chaos sets in, leading to quantum ergodicity of the computer eigenstates. In this regime the noninteracting qubit structure disappears, the eigenstates become complex and the operability of the computer is destroyed. Despite the fact that the spacing between multi-qubit states drops exponentially with the number of qubits $n$, we show that the quantum chaos border decreases only linearly with $n$. This opens a broad parameter region where the efficient operation of a quantum computer remains possible.
\end{abstract}

PACS numbers: 03.67.Lx, 05.45.Mt, 24.10.Cn

Since the pioneering work of Feynman $[1]$ and modern developments of efficient algorithms $[2]$ and errorcorrecting codes [3, 何, the realization of quantum computers became a challenge of modern physics [5]. Different experimental realizations have been proposed, including ion traps [6], nuclear magnetic resonance systems [7], nuclear spins with interaction controlled electronically [8,9], quantum dots [10], Cooper pair boxes [11] and optical lattices 12. A key common feature of these experimental settings is the presence of interacting qubits (two-level systems). Here we analyze the effect of qubit interaction on operability of the quantum computer. The interaction is required since a quantum computer needs to perform two-qubit logical operation such as XOR [5]. We note that such a two-qubit gate has been experimentally realized 13].

In an isolated system of $n$ uncoupled qubits, the dimension of the total Hilbert space $N_{H}$ increases exponentially with $n\left(N_{H}=2^{n}\right)$, while all eigenvalues of the Hamiltonian are included in an energy interval of size $\Delta E \sim n \Delta_{0}$, where $\Delta_{0}$ is the average energy distance between the two states of one qubit. As a result, the average spacing $\Delta_{n}$ between adjacent energy levels of the Hamiltonian decreases exponentially with the number of qubits $\left(\Delta_{n} \sim n \Delta_{0} / N_{H} \ll \Delta_{0}\right)$. When a coupling $J$ between the qubits is added $\left(J<\Delta_{0}\right)$, one still has $\Delta E \sim n \Delta_{0}, N_{H}$ is unchanged, and the above estimate for $\Delta_{n}$ still holds. This general result for $\Delta_{n}$ is related to the exponentially large size $N_{H}$ of the Hilbert space, which is one of the main reasons of the striking efficiency of quantum computing [1,22. It implies that dense highly excited states are needed for the computation. However, when performing the computation one wants to operate with noninteracting multi-qubit states $\left|\psi_{i}\right\rangle=\left|\alpha_{1}, \ldots, \alpha_{n}\right\rangle$ where $\alpha_{k}=0,1$ marks the polarization of each individual qubit. These quantum register states should remain well-defined in the presence of interqubit coupling even if multi-qubit levels are exponentially dense. Therefore the mixing of noninteracting multi-qubit states induced by the interaction is crucial for the computer operability. In the field of quantum chaos 14,15 it is known that noninteracting states will be eventually mixed by the interaction and quantum ergodicity will set in: each quantum computer eigenstate will be composed of a large number of noninteracting multi-qubit states $\mid \psi_{i}>$ and the original quantum register states will be washed out. At first glance one would expect that such mixing happens when the coupling between qubits becomes comparable to the multi-qubit spacing $\Delta_{n}$. In such a case, the creation of quantum computers competitive with classical ones would be rather difficult: since hundreds of qubits are necessary, this would lead to absurdly strict restrictions on coupling strength. Indeed, for $n=1000$, the minimum number of qubits for which Shor's algorithm becomes useful [5], the multi-qubit spacing becomes $\Delta_{n} \sim 10^{3} \times 2^{-10^{3}} \Delta_{0} \sim 10^{-298} \mathrm{~K}$, where we used $\Delta_{0} \sim 1 \mathrm{~K}$ that corresponds to the typical one-qubit spacing in the experimental proposals [8,9]. It is clear that the residual interaction $J$ between qubits in any experimental realization of the quantum computer will be larger than this. For example, in the proposal [9], the increase of effective electron mass by a factor of two, induced by the electrostatic gate potential, means that the spin-spin interaction is changed from $J \sim \Delta_{0} \sim 1 \mathrm{~K}$ (corresponding to a distance between donors of $200 \AA$ and an effective Bohr radius of $30 \AA$ in Eq.2 of [9]) to the residual interaction $J \sim 10^{-5} \mathrm{~K} \gg \Delta_{n}$.

However the problem is not so simple, since the interaction is always of two-body nature and not all of the multi-qubit states are directly coupled. Actually the number of states directly coupled to such a quantum register state $\left|\psi_{i}\right\rangle$ increases not faster than quadratically with $n$. A similar problem appears in other physical many-body interacting systems such as nuclei, complex atoms, quantum dots and quantum spin glasses [16 20]. It was realized that sufficiently strong interaction leads to quantum chaos and internal (dynamical) thermalization, where the eigenstates properties follow the predictions of Random Matrix Theory (RMT) 14 18]. The quantum chaos border for this dynamical thermalization has been 
established only recently and it has been shown that the relevant coupling strength should be larger than the energy spacing between directly coupled states $\Delta_{c}$ [17,20]. Since $\Delta_{c}$ drops algebraically with $n$, it is exponentially larger than $\Delta_{n} \sim n 2^{-n} \Delta_{0}$, and therefore a relatively large coupling strength is required for the emergence of quantum chaos and ergodicity. A similar border for interacting qubit systems would allow a reasonable regime of operability for quantum computers.

To investigate the emergence of quantum chaos in quantum computers, we chose a model of $n$ qubits on a two-dimensional lattice with nearest-neighbour interqubit coupling. The Hamiltonian reads:

$$
H=\sum_{i} \Gamma_{i} \sigma_{i}^{z}+\sum_{i<j} J_{i j} \sigma_{i}^{x} \sigma_{j}^{x}
$$

where the $\sigma_{i}$ are the Pauli matrices for the qubit $i$ and the second sum runs over nearest-neighbour qubit pairs with periodic boundary conditions applied. The energy spacing between the two states of a qubit is represented by $\Gamma_{i}$ randomly and uniformly distributed in the interval $\left[\Delta_{0}-\delta / 2, \Delta_{0}+\delta / 2\right]$. The parameter $\delta$ gives the width of the distribution near the average value $\Delta_{0}$ and varies from 0 to $\Delta_{0}$. Here $\Gamma_{i}$ can be viewed as the splitting of nuclear spin levels in a local magnetic field, as it is discussed in the experimental proposals [8, [9]. The different values of $\Gamma_{i}$ are needed to prepare a specific initial state by electromagnetic pulses in nuclear magnetic resonance. In this case the couplings $J_{i j}$ will represent the hyperfine interaction between the spins, which is needed to build the quantum computer. Different physical mechanisms can generate these couplings, such as spin exciton exchange [8,9], dipole-dipole interaction, etc... For generality we chose $J_{i j}$ randomly distributed in the interval $[-J, J]$. The Hamiltonian (1) can be considered as a generic quantum computer model, which catches the main physics of different experimental proposals. For example a similar Hamiltonian appears in a quantum computer based on optical lattices 12,21]. We restrict ourselves to the case of static couplings which are always present as a residual interaction and are much larger than the multi-qubit spacing $\Delta_{n}$ even for moderate values of $n$. In a sense (1) describes the hardware of the computer, while gates operation in time requires additional studies, which are possible only if the properties of the hardware are well understood.

As is well known in the field of quantum chaos, the transition to ergodic eigenstates is reflected in the level spacing statistics $P(s)$, which goes from the Poisson distribution $P_{P}(s)=\exp (-s)$ for nonergodic states to the Wigner-Dyson (WD) distribution $P_{W}(s)=$ $(\pi s / 2) \exp \left(-\pi s^{2} / 4\right)$, corresponding to RMT, for ergodic states. Here $s$ is the nearest level spacing measured in units of average spacing and $P(s)$ is the probability to find two adjacent levels whose spacing is in $[s, s+d s]$.

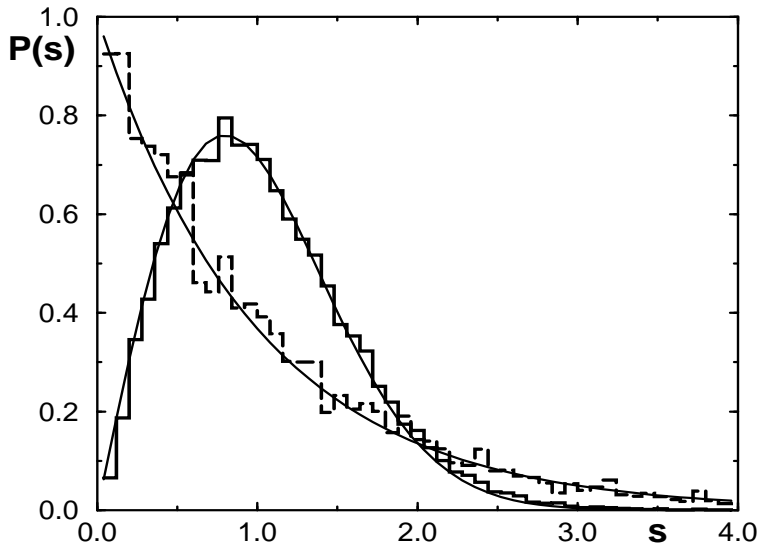

FIG. 1. Transition from Poisson to WD statistics in the model (11) for the states in the middle of the energy band $( \pm 6.25 \%$ around the center $)$ for $n=12$ : $J / \Delta_{0}=0.02, \eta=1.003$ (dashed line histogram); $J / \Delta_{0}=0.48, \eta=0.049$ (full line histogram). Full curves show $P_{P}(s)$ and $P_{W}(s) ; N_{S}>2.5 \times 10^{4}, N_{D}=100, \delta=\Delta_{0}$.

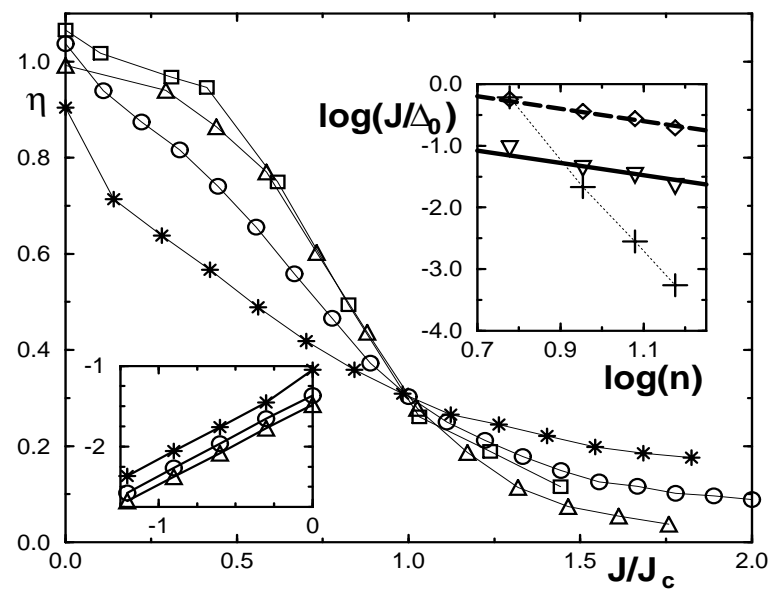

FIG. 2. Dependence of $\eta$ on the rescaled coupling strength $J / J_{c}$ for the states in the middle of the energy band for $n=6(*), 9(\mathrm{o}), 12$ (triangles), 15 (squares); $\delta=\Delta_{0}$. The upper insert shows $\log \left(J_{c} / \Delta_{0}\right)$ (diamonds) and $\log \left(J_{c s} / \Delta_{0}\right)$ (triangles) versus $\log (n)$; the variation of the scaled multi-qubit spacing $\Delta_{n} / \Delta_{0}$ with $\log (n)$ is shown for comparison $(+)$. Dashed line gives the theoretical formula $J_{c}=C \Delta_{0} / n$ with $C=3.16$; the solid line is $J_{c s}=0.41 \Delta_{0} / n$. The lower insert shows $\log \left(J_{c s} / \Delta_{0}\right)$ versus $\log \left(\delta / \Delta_{0}\right)$ for $n=6(*), 9$ (o), 12 (triangles); straight lines have slope 1.

The majority of our data are displayed for the middle of the energy spectrum, where the transition starts, and which therefore sets the limit of operability of the quantum computer. The model (11) has two symmetry classes characterized by an odd or even number of qubits up, and the data are given for one symmetry class. In order to reduce statistical fluctuations, we use $5 \leq N_{D} \leq 4 \times 10^{4}$ random realizations of $\Gamma_{i}$ and $J_{i j}$, as is done usually in 
RMT [15]. Eigenvalues and eigenvectors are computed by exact diagonalization of the Hamiltonian matrix (11) for each realization. In this way the total number of spacings is $10^{4}<N_{S} \leq 1.6 \times 10^{5}\left(N_{S} \propto N_{D} N_{H}\right)$. An example of the transition in the spectral statistics is shown in Fig.1.

To analyze the evolution of $P(s)$ with the coupling $J$, it is convenient to use the parameter $\eta=\int_{0}^{s_{0}}(P(s)-$ $\left.P_{W}(s)\right) d s / \int_{0}^{s_{0}}\left(P_{P}(s)-P_{W}(s)\right) d s$, where $s_{0}=0.4729 \ldots$ is the intersection point of $P_{P}(s)$ and $P_{W}(s)$. In this way $P_{P}(s)$ corresponds to $\eta=1$, and $P_{W}(s)$ to $\eta=0$. As is usual in the field of quantum chaos, the variation of $\eta$ characterizes the evolution of $P(s)$ [20]. The variation of $\eta$ with respect to $J / \Delta_{0}$ is presented in Fig. 2 for $\delta=\Delta_{0}$ showing that indeed $\eta$ drops from 1 to 0 with increasing coupling strength. The transition appears to become sharper for larger system sizes. The typical $J_{c}$ value near which the transition takes place corresponds to intermediate values of $\eta$. We chose the condition $\eta\left(J_{c}\right)=0.3$. The dependence of $J_{c}$ on $n$ is given in the Fig.2. In analogy with other many-body systems discussed in [17,20], we expect that $J_{c} \approx \Delta_{c} \approx C \Delta_{0} / n$, where $C$ is some numerical constant. Indeed, one multi-qubit state is coupled to $2 n$ other states in an energy interval of order $6 \Delta_{0}$. This theoretical estimate is in agreement with the data of Fig.2, with $C \approx 3$. We stress that this critical coupling is exponentially larger than the multi-qubit level spacing $\Delta_{n} \sim n 2^{-n} \Delta_{0}$, as is shown on Fig.2. For the case $\delta \ll \Delta_{0}$, the total spectrum at $J=0$ is composed of $n$ bands with interband distance $2 \Delta_{0}$ and a bandwidth of $\sqrt{n} \delta$. Within one band, one multi-qubit state is coupled to about $n$ states in an energy interval of $2 \delta$, so that $J_{c} \approx \Delta_{c} \sim \delta / n$. This quantum chaos border is still much bigger than $\Delta_{n} \sim \sqrt{n} \delta /\left(N_{H} / n\right) \sim n^{3 / 2} 2^{-n} \delta$.

The transition in the level statistics reflects the drastic change in the multi-qubit structure of the eigenstates of (1). Indeed, Fig.3 shows that for $J<J_{c}$ one eigenstate is formed only by one or few noninteracting states $\left|\psi_{i}\right\rangle$, while for $J>J_{c}$ a huge number of them are required. In the latter case, the computer eigenstates become a random mixture of quantum register states $\left|\psi_{i}\right\rangle$, making rather difficult to perform computation.

To study this drastic change in the structure of eigenstates, it is convenient to use the quantum eigenstate entropy $S_{q}$, defined by: $S_{q}=-\sum_{i} W_{i} \log _{2} W_{i}$, where $W_{i}$ is the quantum probability to find the noninteracting multi-qubit state $\mid \psi_{i}>$ in the eigenstate $\mid \phi>$ of (1) $\left.\left.\left(W_{i}=\left|<\psi_{i}\right| \phi\right\rangle\right|^{2}\right)$. In this way $S_{q}=0$ if $\mid \phi>$ is one noninteracting state $(J=0), S_{q}=1$ if $|\phi\rangle$ is equally composed of two $\left|\psi_{i}\right\rangle$, and the maximal value is $S_{q}=n$ if all $2^{n}$ states contribute equally to $|\phi\rangle$. The variation of the average quantum entropy with $J$ is shown on Fig.4 for $\delta=\Delta_{0}$. It shows that $S_{q}$ grows with $J$ and the transition to ergodic states with large $S_{q}$ takes place in the vicinity of $J_{c}$. In addition these data show that the critical coupling $J_{c s}$ at which $S_{q}=1$ is $J_{c s} \approx 0.13 J_{c}$. The ratio $J_{c s} / J_{c}$ stays within $15 \%$ of the average value when $n$ changes from 6 to 15 , while the ratio $\Delta_{n} / J_{c}$ varies from 1 to $3 \times 10^{-3}$ (see upper insert of Fig.2). The dependence of $J_{c s}$ on $\delta$ is shown on the lower insert of Fig.2; it clearly shows the linear decrease of $J_{c s}$ with $\delta$ and can be well described by $J_{c s}=0.4 \delta / n$. Naturally, the quantum chaos border drops to zero with $\delta$ due to the quasidegeneracy inside the energy bands at $J=0$.

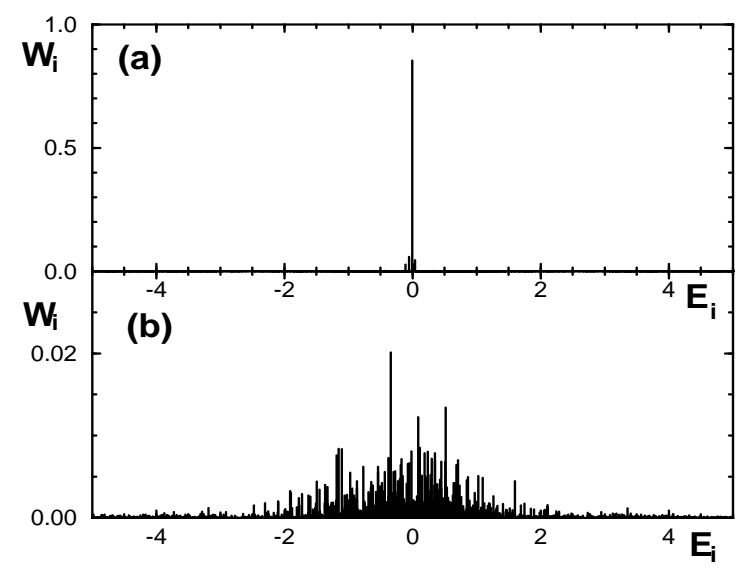

FIG. 3. Two quantum computer eigenstates of model (11) in the basis of noninteracting multi-qubit states, i.e. $W_{i}=\left|\left\langle\psi_{i} \mid \phi\right\rangle\right|^{2}$ as a function of noninteracting multi-qubit energy $E_{i}$ for $n=12$ and $\delta=\Delta_{0}$ with $J_{c} / \Delta_{0}=0.273$ (see text): (a) $J / \Delta_{0}=0.02$; (b) $J / \Delta_{0}=0.48$.

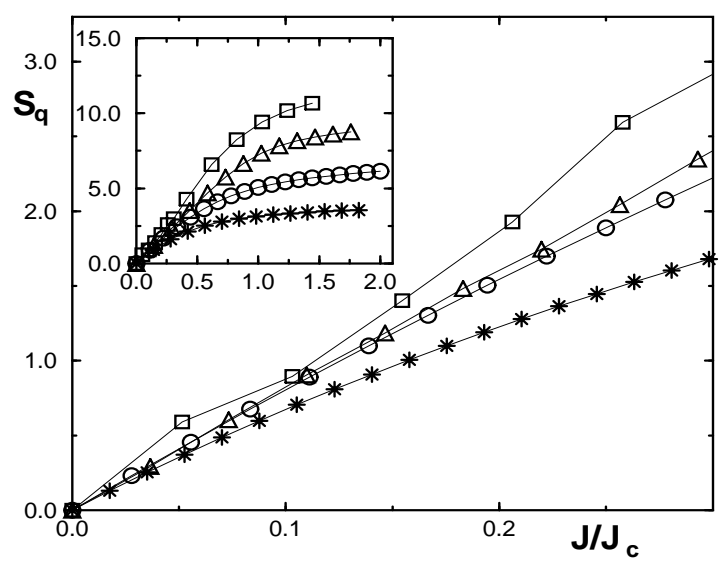

FIG. 4. Dependence of the quantum eigenstate entropy $S_{q}$ on $J / J_{c}$ for $\delta=\Delta_{0}$ and $n=6(*), 9$ (o), 12 (triangles), 15 (squares); $10^{4}<N_{S} \leq 1.6 \times 10^{5}$. Insert shows the dependence on larger scale.

We note that for $n=1000$ and $\delta=\Delta_{0}=1 \mathrm{~K}$, only two multi-qubit states will be mixed at $J_{c s} \approx 0.4 \Delta_{0} / n \approx 0.4$ $\mathrm{mK}$. This critical coupling is much larger than the multiqubit level spacing $\Delta_{n} \sim 10^{-298} \mathrm{~K}$. Even if the quantum border $J_{c s}$ corresponds to a relatively low coupling strength it seems reasonable that the residual interaction between qubits can stay below this threshold with 
current technologies (but not below $\Delta_{n}$ ).

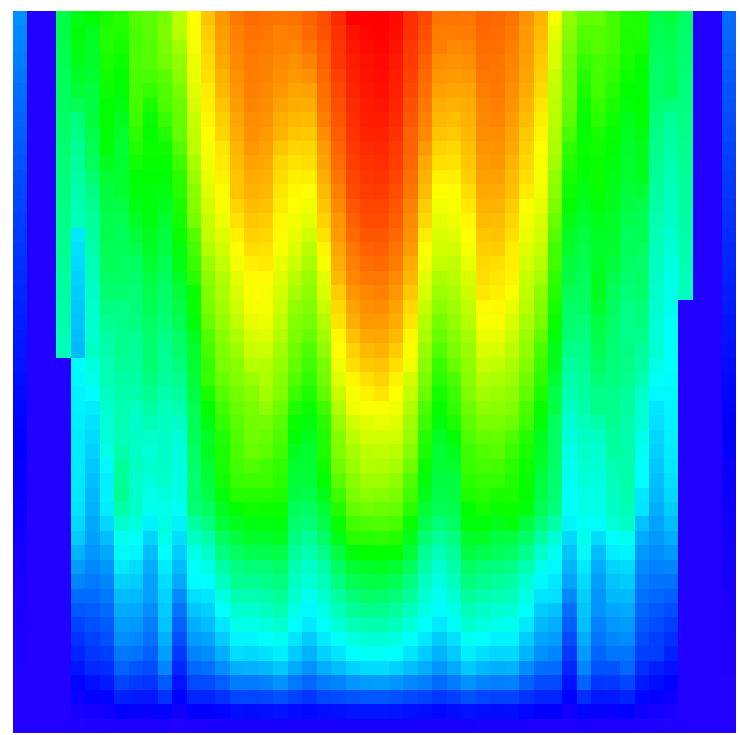

FIG. 5. The quantum computer melting induced by the coupling between qubits. Color represents the level of quantum eigenstate entropy $S_{q}$, from bright red $\left(S_{q} \approx 11\right)$ to blue $\left(S_{q}=0\right)$. Horizontal axis is the energy of the computer eigenstates counted from the ground state to the maximal energy $\left(\approx 2 n \Delta_{0}\right)$. Vertical axis is the value of $J / \Delta_{0}$, varying from 0 to 0.5 . Here $n=12, \delta=\Delta_{0}, J_{c} / \Delta_{0}=0.273$, and one random realization of (1) is chosen.

The pictorial image of the quantum computer melting under the influence of the interqubit coupling $J$ is shown on Fig.5. The melting starts in the middle of the spectrum (high energy) and progressively invades low-energy states and the whole computer, destroying its operability. We stress that this destruction takes place in an isolated system without any external decoherence process. Nevertheless the thermalization in this closed system, which appears because of the interqubit coupling, can mimic the effect of a coupling with the external world and external decoherence. Above the quantum chaos border an initial register state $\mid \psi_{i}>$ will spread quickly with time [22] over an exponential number of eigenstates of the system with residual interaction, destroying gates operability.

Our studies show that the mixing of multi-qubit states and onset of quantum chaos induced by interqubit coupling leads to the melting of a realistic quantum computer and destruction of its operability; however, the quantum chaos border found for this process corresponds to a relatively strong interaction, being exponentially larger than the energy level spacing between multi-qubit states. We expect that below this border, error-correcting codes [3, 4 , will allow to perform efficient quantum computing with a large number of qubits. However we note that quantum chaos sets in very easily if the fluctuation amplitude $\delta$ of individual qubit spacing drops to zero $\left(J_{c} \propto \delta\right)$.
We thank O.P. Sushkov and I.D. Vagner for stimulating discussions, and the IDRIS in Orsay and the CICT in Toulouse for access to their supercomputers.

[1] R. P. Feynman, Found. Phys. 16, 507 (1986).

[2] P. W. Shor, in Proc. 35th Annu. Symp. Foundations of Computer Science (ed. Goldwasser, S. ), 124 (IEEE Computer Society, Los Alamitos, CA, 1994).

[3] A. R. Calderbank and P. W. Shor, Phys. Rev. A 54, 1098 (1996).

[4] A. Steane, Proc. Roy. Soc. Lond. A 452, 2551 (1996).

[5] A. Steane, Rep. Progr. Phys. 61, 117 (1998).

[6] J. I. Cirac and P. Zoller, Phys. Rev. Lett. 74, 4091 (1995).

[7] N. A. Gershenfeld and I. L. Chuang, Science 275, 350 (1997); D. G. Cory, A. F. Fahmy and T. F. Havel, In Proc. of the 4th Workshop on Physics and Computation (Complex Systems Institute, Boston, MA, 1996).

[8] V. Privman, I. D. Vagner and G. Kventsel, Phys. Lett. A 239, 141 (1998).

[9] B. E. Kane, Nature 393, 133 (1998).

[10] D. Loss and D. P. Di Vincenzo, Phys. Rev. A 57, 120 (1998).

[11] Y. Nakamura, Yu. A. Pashkin, and J. S. Tsai, Nature 398, 786 (1999).

[12] G. K. Brennen, C. M. Caves, P. S. Jessen and I. H. Deutsch Phys. Rev. Lett. 82, 1060 (1999); D. Jaksch, H. J. Briegel, J. I. Cirac, C. W. Gardiner and P. Zoller, Phys. Rev. Lett. 82, 1975 (1999).

[13] C. Monroe, D. M. Meekhof, B. E. King, W. M. Itano and D. J. Wineland, Phys. Rev. Lett. 75, 4714 (1995).

[14] Les Houches Lecture Series 52, Eds. Giannoni, M.-J., Voros, A. and Zinn-Justin, J. (North-Holland, Amsterdam, 1991).

[15] T. Guhr, A. Müller-Groeling and H. A. Weidenmüller, Phys. Rep. 299, 189 (1999).

[16] J. B. French and S. S. M. Wong, Phys. Lett. B 33, 449 (1970); O. Bohigas and J. Flores, ibid 34, 261 (1971).

[17] S. Åberg, Phys. Rev. Lett. 64, 3119 (1990).

[18] V. Zelevinsky, B. A. Brown, N. Frazier and M. Horoi, Phys. Rep. 276, 85 (1996); V. V. Flambaum, F. M. Izrailev, and G. Casati, Phys. Rev. E 54, 2136 (1996).

[19] U. Sivan, F. P. Milliken, K. Milkove, S. Rishton, Y. Lee, J. M. Hong, V. Boegli, D. Kern, and M. de Franza, Europhys. Lett. 25, 605 (1994).

[20] D. L. Shepelyansky and O. P. Sushkov, Europhys. Lett. 37, 121 (1997); P. Jacquod and D. L. Shepelyansky, Phys. Rev. Lett. 79, 1837 (1997); A. D. Mirlin and Y. V. Fyodorov, Phys. Rev. B 56, 13393 (1997); D. Weinmann, J.-L. Pichard and Y. Imry, J. Phys. I France 7, 1559 (1997); B. Georgeot and D. L. Shepelyansky, Phys. Rev. Lett. 81, 5129 (1998).

[21] A. Sørensen and K. Mølmer, Phys. Rev. Lett. 83, 2274 (1999).

[22] As in many-body systems [20 the spreading rate $\gamma$ can be estimated as $\gamma \sim J^{2} / \Delta_{c} \sim n J^{2} / \delta$ for $J_{c}<J<\delta$. 